\title{
Genotype and environment interaction of drought tolerant rice genotypes evaluated in drought prone areas of Bangladesh
}

\author{
R. R. Majumder*, T. K. Hore, M. A. Kader and T. L. Aditya \\ Plant Breeding Division, Bangladesh Rice Research Institute, Gazipur-1701, Bangladesh \\ *E-mail: rrmajumder@yahoo.com.
}

\begin{abstract}
Field trials were conducted using ten rice genotypes including four national and international check varieties under drought prone rainfed environments of Rangpur, Nilphamari, Kurigram and Lalmonirhat districts of Bangladesh in Transplant Aman 2012 season to evaluate the growth duration and grain yield stability as well as adaptability. The experiment used randomized complete block design with three replications. Growth duration and Grain yield data were subjected to Additive Main effects and Multiplicative Interaction (AMMI) analysis. The mean growth duration of 10 genotypes over four environments showed that the longest growth duration was found for IR83383-B-B-129-4 (115.6 days) and the lowest growth duration was found for BRRI dhan56 (Ck) (113.4 days). IR82635-B-B-145-1 produced maximum 4.28 t/ha grain yield followed by 4.28 t/ha in IR82589-B-B-84-3 and the lowest grain yield was recorded for IR83376-B-B-130-2 with 3.24 t/ha. It was evident from the AMMI 1 biplot analysis that IR83377-B-B-933, IR83383-B-B-129-4, IR82635-B-B-145-1, IR82635-B-B-75-2 and BRRI dhan56 (Ck) generally exhibited high yield with high main (additive) effects showing positive first principal component of the interaction (IPCA1), but IR82635-B-B-145-1 being the overall best. Hence, IR82635-B-B-145-1 was identified as specially adapted to Lalmonirhat and this environment was considered as the wide range suitable environment for the genotype. Lalmonirhat could be regarded as a good selection site for rice improvement due to stable yields. IR83377-B-B-93-3, IR82635-B-B-145-1, IR83383-B-B-129-4, IRRI 123 (Ck), BRRI dhan57 (Ck) and BINA dhan7 (Ck) were hardly affected by the $G \times E$ interaction and would perform well across a wide range of environments. In AMMl 2 biplot, IR82635-B-B-145-1 and IRRI 123 (Ck) was more responsive since they were away from the origin whereas other genotypes were nearer to the origin and hence they were more stable to different environments. IR83383-B-B-129-4 was very close to the origin, so it was more stable to different environments. Similarly Rangpur Sadar and Lalmonirhat showed similar potentiality over IR83383-B-B-129-4. The study it revealed that IR83377-B-B-93-3, IR83383-B-B-129-4, IR82635-B-B-145-1, IR82635-B-B-75-2 and IR82589-B-B-84-3 has the potentiality to show drought tolerance for the regions and might be suitable for releasing as a variety after conducting proper yield evaluation trials and Distinction, Uniformity and Stability (DUS) tests.
\end{abstract}

Keywords: Rice, Drought tolerant, Genotypes, Environment, Yield, AMMI analysis

\section{Introduction}

Rice (Oryza sativa L.) is the staple food of more than three billion people in the world and a major source of global food calories. Drought is a major yield limiting factor affecting the crop productivity worldwide (Hussain et al., 2011). Drought in Bangladesh affect about 2.32 and 1.2 million hectare of cropped land annually during the Aman (July to October) and Boro (November to June) seasons, respectively (Ibrahim, 2001). Drought is a recurrent phenomenon and an important constraint on agricultural production in humid and sub humid rice-growing areas of Asia. At least 23 million hectares in Asia (20 per cent of the total rice area) are subject to drought of varying intensities, which is one of the major factors contributing to low and unstable production in Bangladesh. The economic costs of drought include not only the rice production loss, but also the loss in production of post-rice crops grown on residual soil moisture. Drought in the context of agriculture is a situation when the water availability to plant is less than what is required to sustain its growth and development (Deikman et al., 2011). Drought tolerance is the ability of the plant to survive in water limited conditions (Turner, 1979). Karim et al., (1990) mentioned that due to uncertain rain and its uneven distribution in Bangladesh, drought of different intensities occurs during the growing period of T. Aman. Islam (1996) stated that the farmers cultivate medium duration Aman varieties in the region. Early drought delays transplanting of Aman and as a result shifts the harvesting time. Consequently, the Aman rice, on the other hand, is also affected due to drought during the reproductive stage. Thus sustainable food self-sufficiency of Bangladesh is being threatened by devastating drought. Increasing population pressure, unpredictable rainfall patterns, shrinking fresh water resources, and increased frequency of severe drought spells in recent years are the reasons behind putting concerted efforts toward breeding drought-tolerant rice cultivars much needed by the rice farmers. Drought 
mitigation through development of drought resistant varieties with higher yields suitable for water-limiting environments will be a key to improve rice production and ensure food security to 3 billion people in Asia. This study encompasses an overview of the activities in rice emphasizing the adaption of the drought tolerant genotypes in drought prone regions of Bangladesh and to identify best genotype(s) that could be able to alleviate drought in Bangladesh.

\section{Materials and Methods}

Ten promising drought tolerant rainfed rice genotypes including four national and international drought tolerant check varieties were grown in four locations of Rangpur Sadar, Nilphamari, Lalmonirhat and Kurigram of Bangladesh during T. Aman 2012 season. Twenty one to twenty four days old seedlings were transplanted @ 2-3 seedlings per hill at $20 \mathrm{~cm} \times 20 \mathrm{~cm}$ spacing in Randomized Complete Block Design with 3 replications in four districts. Fertilizers @ 69 (152 Kg Urea): 10 (52 Kg TSP): 41 (82 Kg MP): 11 (60 Kg Gypsum): 4 (11 Kg Zn SO 4$) \mathrm{Kg} / \mathrm{ha}$ NPKSZn were applied in the trial. All amounts of $\mathrm{P}, \mathrm{K}$, $\mathrm{S}$ and $\mathrm{Zn}$ were applied at the time of final land preparation and nitrogen was applied at three equal splits at 10, 25-30 and 40-45 days after transplanting (BRRI, 2015). Crop management such as weeding, irrigation etc. was done in time. Insects, diseases and other pests were controlled properly. Drought stress was initiated four weeks after transplanting up to maturity through proper draining of water from the field. Growth duration and grain yield data were collected from 4 environments. Data were analyzed following ANOVA model for single site experiment conducted in randomized complete block design (Gomez and Gomez, 1984) as given below

$Y i j=\mu+G i+B j+e i j$

where $Y \mathrm{ij}=$ observed phenotype of ith genotype in $j$ th block, $\mu=$ overall mean, $\mathrm{Gi}=$ effect of $i$ th genotype, $\mathrm{Bj}=$ effect of $j$ th block, eij= effect of experiment error. Error variances for each traits were test for variance homogeneity using Bartlett's test (Snedecor and Cochran, 1983), and data the traits with homogenous variances were combined.

Finally Yijk $=\mu+G i+E j+B j / k+(G x E) i j+$ eijk was used to analyse cross site variance components. where Yijk= observed phenotype of ith genotype in $k$ th block under $j$ th environment, $\mu=$ overall mean, $\mathrm{Gi}=$ effect of ith genotype, Ej= effect of jth environment, $\mathrm{Bj} / \mathrm{k}=$ effect of $k$ th block under $j$ th environment, $(G \times E) i j=$ effect of interaction between ith genotype and jth environment, eijk= effect of experiment error. ANOVA and Genotype-Environment Interaction (GEI) were estimated by the AMMI model (Zobel et al., 1988) using Cropstat version 7.2 software.

\section{Results and Discussion}

\section{Analysis of Variance}

The analysis of variance for growth duration (days) and grain yield (t/ha) of 10 genotypes in four environments is represented in Table 1 and Table 2, respectively. The analysis revealed that variances for genotypes as well as environments were significant but $G \times E$ interaction was not significant in case of growth duration. This implies that there was no significant variation in growth duration among the genotypes over four different environments but there had substantial variation among the genotypes. The main effects of growth duration for genotype explained $40.76 \%$, that of environment accounted explained $37.64 \%$ and for $\mathrm{G} \times \mathrm{E}$ interaction explained $10.63 \%$ of the total variation.

Table 1. Additive Main effects and Multiplicative Interaction analysis of variance for growth duration (days) of the genotypes across environments

\begin{tabular}{lccccc}
\hline Source of Variation & df & SS & MS & F & \% explained \\
\hline Genotypes (G) & 9 & 66.50 & 7.39 & $3.70^{* *}$ & 40.76 \\
Environments (E) & 3 & 61.41 & 20.47 & $10.24^{* *}$ & 37.64 \\
Interaction G × E (GEI) & 27 & 17.34 & 0.64 & 0.32 & 10.63 \\
AMMI COMPONENT1 & 11 & 10.52 & 0.96 & 0.48 & 6.45 \\
AMMI COMPONENT2 & 9 & 5.36 & 0.60 & 0.30 & 3.29 \\
AMMI COMPONENT3 & 7 & 1.45 & 0.21 & 0.10 & 0.89 \\
Error & 80 & 159.86 & 2.00 & & \\
\hline Total & 119 & 163.14 & & & \\
\hline
\end{tabular}


The variance of genotype, environments and $G \times E$ interaction for grain yield were significant along four environments. This implies that there was substantial difference in grain yield among the genotypes in four different environments. The main effects of grain yield for genotype explained $43.35 \%$ and that of environment accounted explained $37.31 \%$ and for $G \times E$ interaction explained $19.12 \%$ of the total variation. The higher sum of squares for genotype indicated that the genotypes were diverse, with higher differences among genotypic means causing most of the variation in grain yield which is in harmony with the findings of Misra et al., (2009) and Fentie et al., (2013) in rice production.

Table 2. Additive Main effects and Multiplicative Interaction analysis of variance for grain yield (t/ha) of the genotypes across environments

\begin{tabular}{lccccc}
\hline Source of Variation & $\mathrm{df}$ & $\mathrm{SS}$ & $\mathrm{MS}$ & $\mathrm{F}$ & \% explained \\
\hline Genotypes (G) & 9 & 4.182 & 0.465 & $7.506^{* *}$ & 43.35 \\
Environment (E) & 3 & 3.599 & 1.200 & $19.381^{* *}$ & 37.31 \\
Interaction G × E (GEI) & 27 & 1.844 & 0.068 & $1.100^{* *}$ & 19.12 \\
AMMI COMPONENT1 & 11 & 0.987 & 0.090 & $1.450^{*}$ & 10.23 \\
AMMI COMPONENT2 & 9 & 0.453 & 0.050 & 0.813 & 4.69 \\
AMMI COMPONENT3 & 7 & 0.404 & 0.058 & 0.933 & 4.19 \\
Error & 80 & 4.952 & 0.062 & & \\
\hline Total & 119 & 9.647 & & & \\
\hline
\end{tabular}

\section{Stability of Growth Duration (days)}

The mean growth duration of 10 genotypes over 4 environments is represented in Table 3 showed that IR83383-B-B-129-4 had the highest (115.6 days) and BRRI dhan57 had the lowest (110.9 days) growth duration. The genotypes showed less consistent performances across all environments. The growth duration of all the genotypes ranged from 108.3 to 117.3 days for all the environments. The average growth duration of all environments for all the genotypes was 114.2 days. On the basis of environmental index value considering negative and positive, Rangpur Sadar (E1) (1.4) was rich environment followed by Nilphamari (E2) (0.5) and Kurigram (E4) (0.2) but Lalmonirhat (E3) (-2.0) was the poor environment (Table 3). Within the genotypes, IR82589-B-B-84-3, BRRI dhan56 (Ck) and BRRI dhan57 (Ck) showed better performance in adverse environment due to their negative phenotypic index.

Table 3. Stability parameters for growth duration (days) of 10 rice genotypes in 4 environments

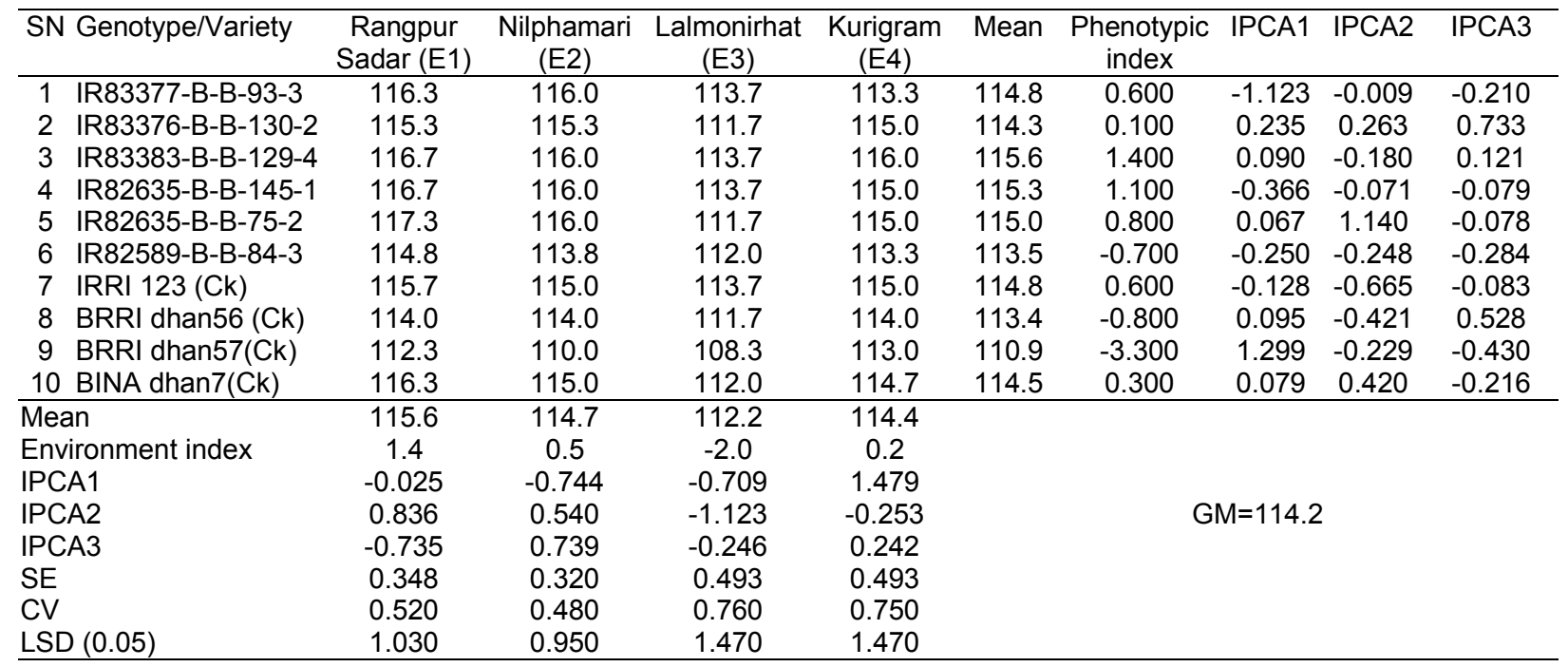




\section{Stability of Grain Yield ( $t / h a)$}

Table 4 shows the mean grain yield over 4 environments. Maximum grain yield was found for IR82635-BB-145-1 (4.28 t/ha) followed by IR82589-B-B-84-3 (4.25 t/ha) and the lowest grain yield was recorded for IR83376-B-B-130-2 (3.24 t/ha). The grain yield of all the genotypes ranged from 3.02 to $4.64 \mathrm{t} / \mathrm{ha}$ in all the environments. The average grain yield of all environments for all the genotypes was $3.82 \mathrm{t} / \mathrm{ha}$. On the basis of environmental index value considering negative and positive, Lalmonirhat (E3) (-0.212) and Kurigram (E4) (-0.33) were poor, Rangpur Sadar (E1) (0.098) was medium and Nilphamari (E2) (0.443) were rich environments. Within the genotypes IR82635-B-B-145-1, IR82589-B-B-84-3, IR82635-B-B-752, IR83377-B-B-93-3, BRRI dhan56 (Ck) and IR83383-B-B-129-4 had higher average yields and negative index values which indicated the genotypes could be adapted to the stress/drought prone environments. IRRI 123 (Ck) also yielded high and due to positive phenotypic index it is suitable for favorable environment. IR83376-B-B-130-2, BRRI dhan57 (Ck) and BINA dhan7 (Ck) were also adapted to favorable environments.

Table 4. Stability parameters for grain yield (t/ha) of 10 rice genotypes in 4 environments

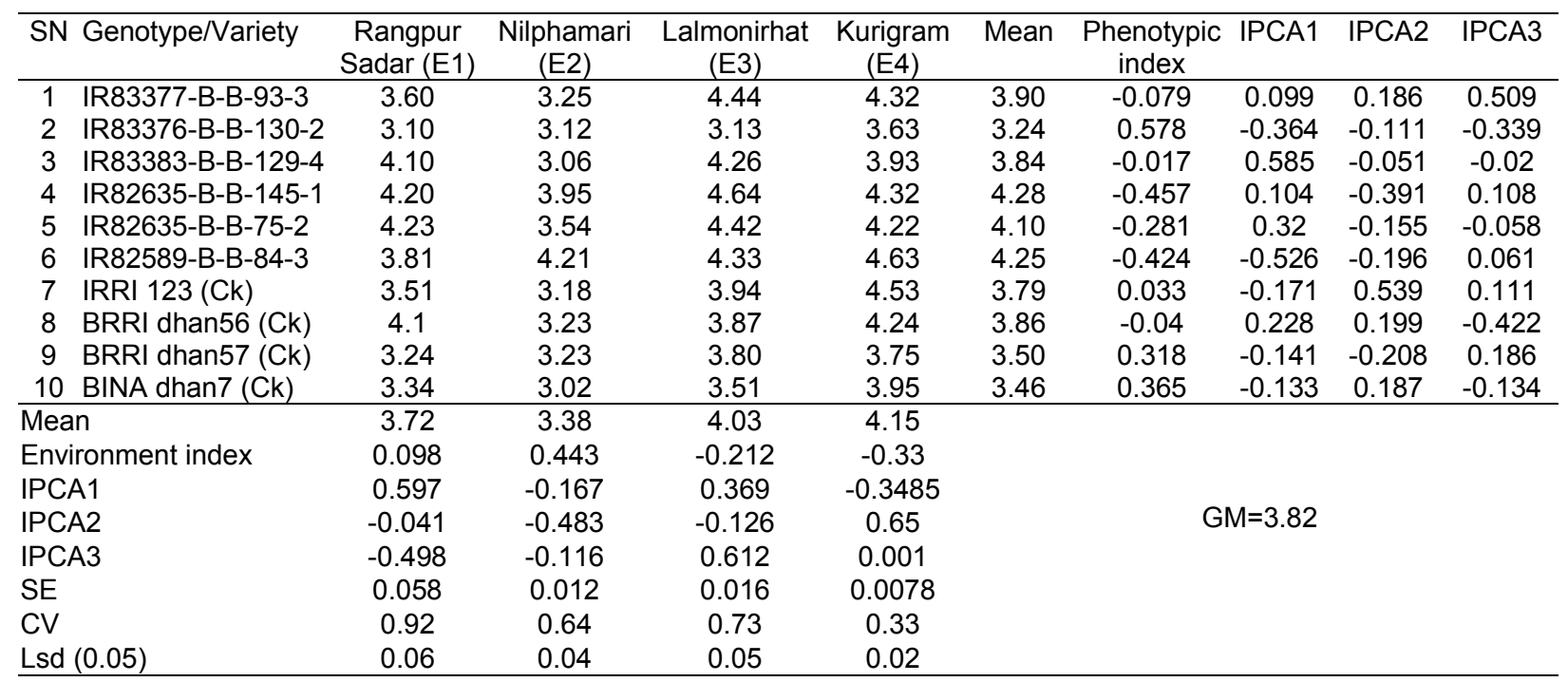

Significant $G \times E$ interaction variance is suggestive of differential performance of varieties under different environments. IR82589-B-B-84-3 out yielded in Nilphamari (E2) and Kurigram (E4) among the genotypes, IR82635-B-B-75-2 out yielded in Rangpur Sadar (E1) where as IR82635-B-B-145-1 out yielded in Lalmonirhat (E3) might be suitable for the specific locations. Farshadfar and Sutka (2003), Asenjo et al., (2003), Das et al., (2010) and Kulsum et al., (2013) analyzed G $\times$ E interaction in rice by AMMl model. They found significant $G \times E$ interaction for grain yield and stated the usefulness of $A M M I$ analysis for selection of genotypes for specific location/environment.

\section{AMMI Analysis}

Biplot analysis is possibly the most powerful tool for AMMI models for visualizing the inter relationships. There are two basic AMMI biplots, the AMMI 1 biplot where the main effects (genotype mean and environment mean) and IPCA1 scores for both genotypes and environments are plotted against each other visualizing the magnitude of interaction for each genotype and each environment. On the other hand, the second biplot is AMMI 2 biplot where scores for IPCA1 and IPCA2 are plotted.

The AMMI-1 biplot for grain yield of 10 genotypes at 4 environmental conditions is presented in Fig. 1. In the AMMI 1 biplot, the usual interpretation of biplot is that the displacements along the abscissa indicate differences in main (additive) effects, whereas displacements along the ordinate indicate differences in interaction effects. Genotypes that group together have similar adaptation while environments which group together influences the genotypes in the same way (Kempton, 1984). The best adapted genotype can plot far from the environment. If a genotype or an environment has an IPCA1 score of nearly zero, it 
has small interaction effects and considered as stable. When a genotype and environment have the same sign on the PCA axis, their interaction is positive and if different, their interaction is negative. The AMMI 1 biplot gave a model fit $91.1 \%$. This result is in agreement with the findings of Nadeem et al., (2007), Gauch and Zobel (1996), Annichiarico (2002) and Zobel (1988). Genotypes and environments on the same parallel line, relative or ordinate have similar yields and a genotype or environment on the right side of the midpoint of this axis has higher yields than those of left hand side. Consequently, among the genotypes IR83377-B-B-93-3, IR83383-B-B-129-4, IR82635-B-B-145-1, IR82635-B-B-75-2 and BRRI dhan56 ( $\mathrm{Ck}$ ) were generally exhibited high yield with high main (additive) effects showing positive IPCA1 score, but the IR82635-B-B-145-1 being the overall best. Hence, IR82635-B-B-145-1 was identified as specially adapted to Lalmonirhat (E3) and this environment was considered as the wide range suitable environment for the genotype. Similar outcome has reported by Das et al., (2010) and Kulsum et al., (2013). Since, Lalmonirhat (E3) had positive IPCA1 score and nearest to zero from other environments and hence had medium interaction effects indicating that all the genotypes performed well in these locations. Thus Lalmonirhat (E3) was considered as the favorable environments for the above mentioned genotypes. IR83377-B-B-93-3 and IR82635-B-B-145-1 showed IPCA1 score close to zero, indicating that these genotypes were stable and less influenced by the environments (Yau, 1995). This result is in agreement with the findings of Adugna (2007) and Anandan et al., (2009). Similarly, Kurigram (E4) was found favorable environment for the genotype IR82589-B-B-84-3 having negative IPCA1 score but with the higher yield than the average yield. Other genotypes showed below average yield. Similarly, IRRI 123 (Ck), BRRI dhan57 (Ck) and BINA dhan7 (Ck) were more stable across environments (low negative IPCA1 score) with below average yield. The genotypes with negative IPCA1 score near zero indicated that these varieties were less influenced by the environments. None of the genotypes were found suitable for the environment Rangpur Sadar (E1). Finally, AMMI 1 biplot statistical model has been used to diagnose the $G \times E$ interaction pattern of grain yield of rice resulting the genotypes IR83377-B-B-93-3, IR82635-B-B-145-1, IR83383-B-B-129-4, IRRI 123 (Ck), BRRI dhan57 (Ck) and BINA dhan7 (Ck) were hardly affected by the $G \times E$ interaction and thus will perform well across a wide range of environments. Lalmonirhat (E3) could be regarded as a good selection site for rice improvement due to more or less stable yields.

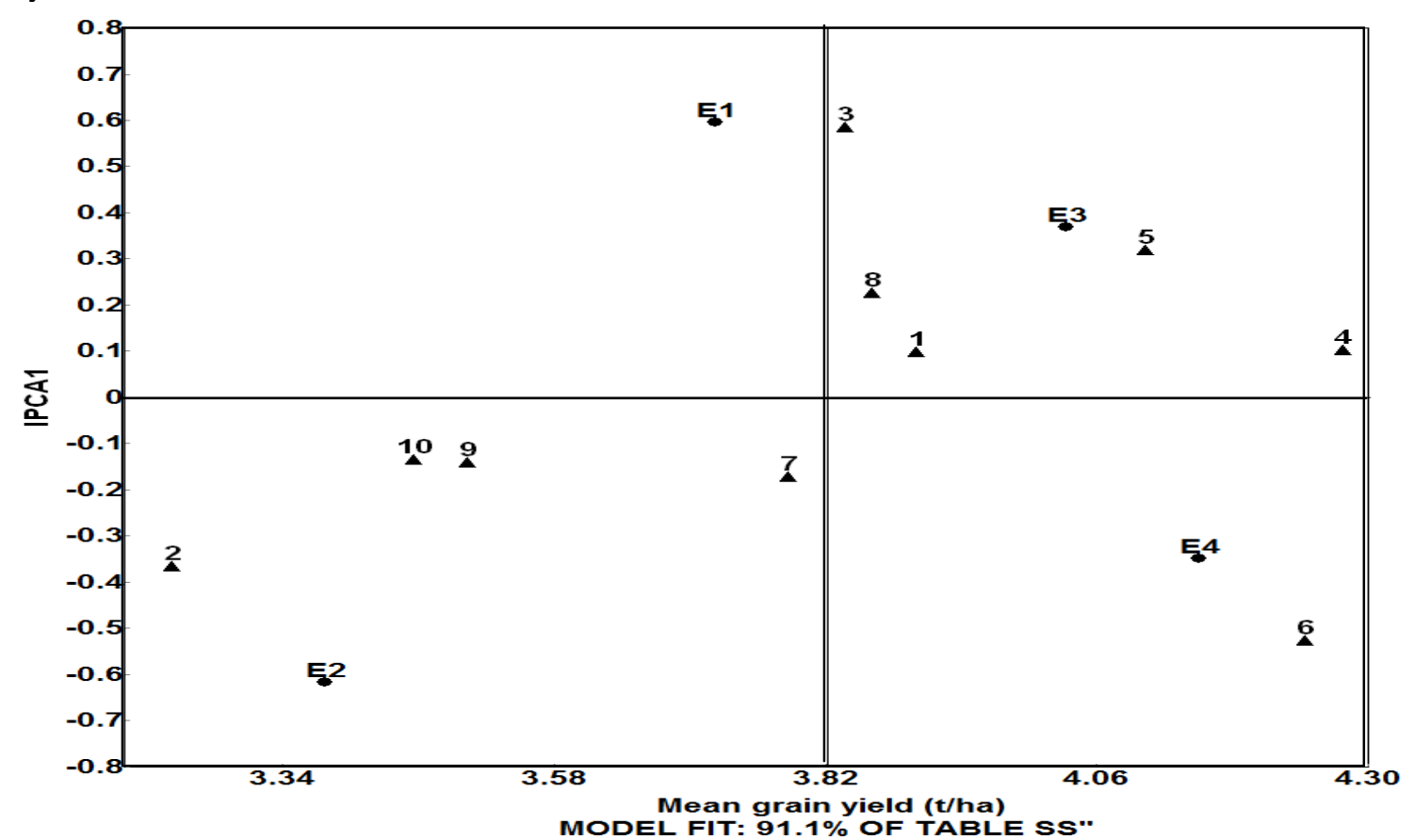

Fig. 1. AMMI 1 Biplot for grain yield (t/ha) of 10 rice genotypes $(G)$ and four environments $(E)$ using genotypic and environmental scores against their respective IPCA1

(First principal component of the interaction $=$ IPCA1, 1=IR83377-B-B-93-3, 2=IR83376-B-B-130-2, 3= IR83383-BB-129-4, 4= IR82635-B-B-145-1, 5= IR82635-B-B-75-2, 6= IR82589-B-B-84-3, 7= IRRI 123 (Ck), 8= BRRI dhan56 (Ck), 9= BRRI dhan57 (Ck) and 10= BINA dhan7 (Ck); E1= Rangpur Sadar, E2= Nilphamari, E3= Lalmonirhat Sadar and $\mathrm{E} 4=$ Kurigram) 
In AMMI 2 biplot, (Fig. 2) the environmental scores are joined to the origin by side lines. Sites with short spokes show little interaction. Those with long spokes exert strong interaction. From the Fig. 2 the points representing Rangpur Sadar (E1), Nilphamari (E2), Lalmonirhat (E3) and Kurigram (E4) are connected to the origin. Rangpur Sadar (E1) and Lalmonirhat (E3) had short spokes and they do not exert strong interaction forces. The genotypes occurring close together on the plot will tend to have similar yields in all environments, while genotypes far apart may either differ in mean yield or show a different pattern of response over the environments. Hence, the genotypes near the origin are more or less stable to the different environmental interaction and those distant from the origins are sensitive and have large interaction. Similarly environment which group together have similar pattern of interaction over the genotypes. Thus the AMMI 2 biplot gave model fit to $78.1 \%$ of $\mathrm{G} \times \mathrm{E}$ interaction. In the present study, the genotype IR82635-B-B-145-1 and IRRI 123 (Ck) had more responsive since they were away from the origin whereas the genotypes IR83377-B-B-93-3, IR83376-B-B-130-2, IR83383-B-B-129-4, IR82635-B-B75-2, IR82589-B-B-84-3, BRRI dhan56 (Ck), BRRI dhan57 (Ck) and BINA dhan7 (Ck) were nearer to the origin and hence they were least sensitive to environmental interaction. IR83383-B-B-129-4 was very close to the origin, so it is stable towards the environments. Here genotype IR83383-B-B-129-4 showed similar potentiality over Rangpur Sadar (E1) and Lalmonirhat (E3). In the same way IR82635-B-B-75-2 showed stability in Lalmonirhat. For multivariate approach, the AMMI model is better for partitioning the $G$ $\times E$ into the causes of variation, which identifies environments' potential and used to identify superior genotypes either with specific adaptation or wide adaptation (Anandan et al., 2009., Crossa, 1990 and Kempton, 1984).

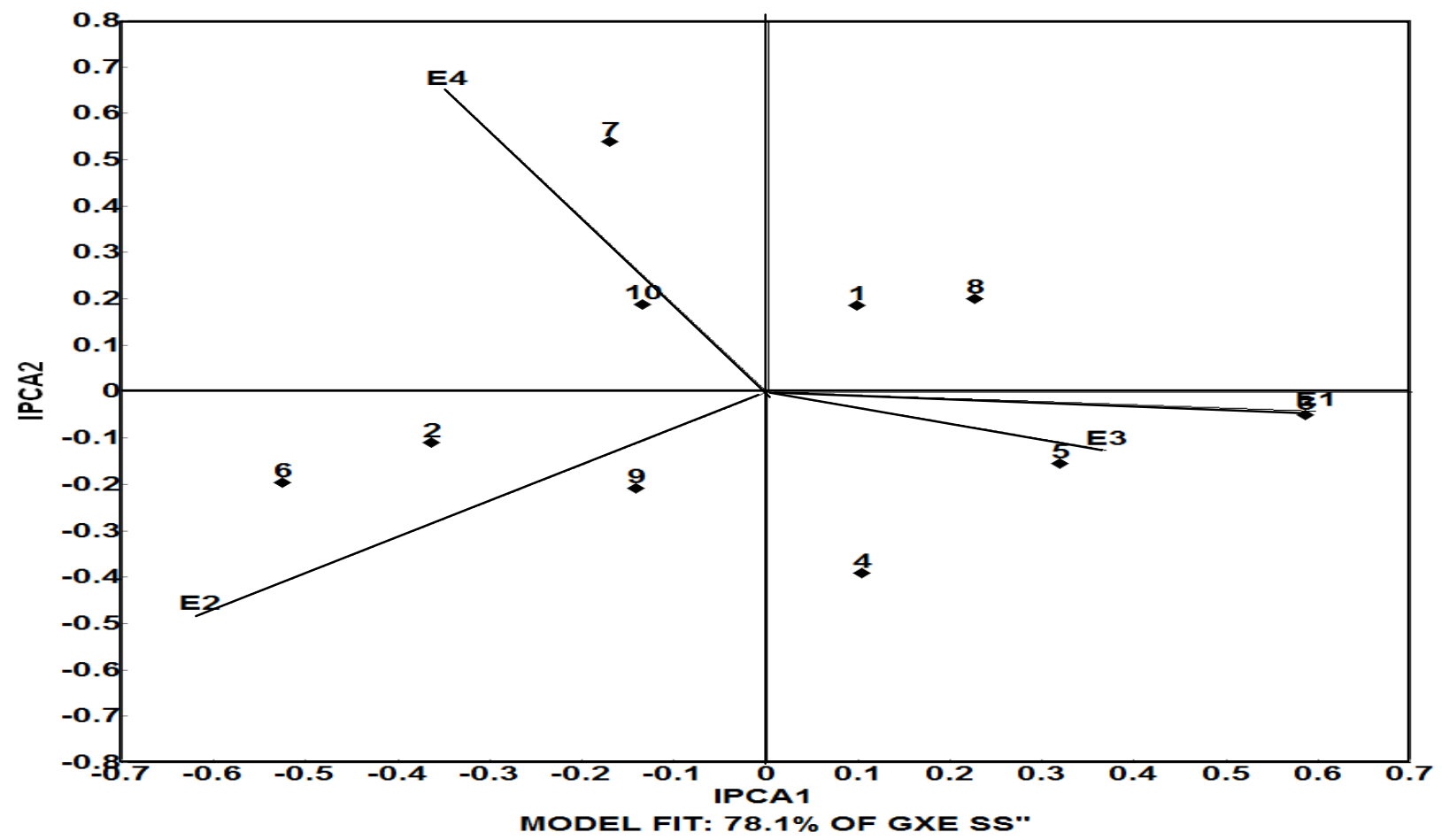

Fig. 2. AMMI 2 model for grain yield (t/ha) of 10 rice genotypes $(G)$ and four environments (E) showing IPCA1 scores at different environments

(First principal component of the interaction $=I P C A 1$, Second principal component of the interaction $=\mid \mathrm{PCA} 2$, $1=I R 83377-B-B-93-3,2=$ IR83376-B-B-130-2, 3= IR83383-B-B-129-4, 4= IR82635-B-B-145-1, 5= IR82635-B-B-75-2, 6= IR82589-B-B-84-3, 7= IRRI 123, 8= BRRI dhan56 (Ck), 9= BRRI dhan57 (Ck) and 10= BINA dhan7 (Ck); E1= Rangpur Sadar, E2= Nilphamari, E3= Lalmonirhat Sadar and E4= Kurigram) 
So from the experiment, it could be summarized that IR83383-B-B-129-4, IR82635-B-B-75-2, IR82589-BB-84-3, IR83377-B-B-93-3 and IR83376-B-B-130-2 were stable genotypes over different environments and therefore could be released as variety after proper yield evaluation trials made by National Seed Board (NSB) of Bangladesh. These drought tolerant genotypes might be also used in the hybridization program to develop new promising drought tolerant breeding lines.

\section{Conclusion}

Crop yield is a complex trait that is influenced by a number of component characters along with the environment directly or indirectly. If we could develop high yielding stable rice variety for diverse environments, we could offer most diverse stable rice varieties for the farmers. AMMI statistical model could be a great tool to select the most suitable and stable high yielding rice varieties for specific as well as for diverse environments. In the present study, AMMI model has shown that the largest proportion of the total variation in grain yield was attributed to different locations. Here most of the genotypes showed environment specificity. The mean grain yield value of genotypes averaged over environments indicated that highest yield was found for IR82635-B-B-145-1 (4.28 t/ha) and the lowest yield was observed for IR83376-B-B-130-2 (3.24 t/ha). It is noted that the genotype IR82635-B-B-145-1 showed higher grain yield (4.64 t/ha) than all other varieties over all the environments. The genotypes IR83377-B-B-93-3, IR83383-B-B-129-4, IR82635-B-B-75-2, IR82589-B-B-84-3, BRRI dhan56 (Ck) and BINA dhan7 (Ck) were hardly affected by the $G \times E$ interaction and thus would perform well across a wide range of environments. As the potential genotypes IR82635-B-B-75-2 and IR82589-B-B-84-3 has already released as varieties for the drought prone regions of Bangladesh, IR82635-B-B-145-1, IR83377-B-B-93-3 and IR83383-B-B-129-4 also have the potentialities to combat in the in different drought prone environments. So these two genotypes could be released as drought tolerant varieties. Hybridization could be also done with these genotypes to generate more stable breeding lines over different environments.

\section{Acknowledgement}

Authors express thanks to the Integrated Agricultural Productivity Project (IAPP)- Bangladesh Rice Research Institute (BRRI) Part, Gazipur for supporting this study through the Ministry of Agriculture (MoA), Government of the People's Republic of Bangladesh in the project activities.

\section{References}

Adugna, A. 2007. Assessment of Yield Stability in Sorghum. African Crop Science Journal. 15(2): 83-92.

Anandan, A., Eswaran, R., Sabesan, T. and Prakash, M. 2009. Additive Main Effects and Multiplicative Interactions Analysis of Yield Performances in Rice Genotypes under Coastal Saline Environments. Advances in Biological Research. 3(1-2): 43-4.

Annichiarico, P. 2002. Genotype × Environment Interactions - Challenges and Opportunities for Plant Breeding and Cultivar Recommendation. Food and Agriculture Organization (FAO), Rome.

Asenjo, C.A., Bezus, R. and Acciaresi, H.A. 2003. Genotype $\times$ environment interaction in rice (Oryza sativa L.) in temperate regions using the Joint Regression Analysis and AMMJ methods. Cereal Research Communications. 32: 97-104.

BBS (Bangladesh Bureau of Statistics). 1994. 1993 Statistical Yearbook of Bangladesh. Dhaka.

BRRI. 2015. Adhunik dhaner chash. (1 ${ }^{\text {th }}$ edn), Bangladesh Rice Research Institute, Gazipur-1700, Bangladesh. p. 23-33.

Cropstat 7.2 for windows tutorial manual. 2009. Crop Research Informatics Laboratory. International Rice Research Institute, pp. 379.

Crossa, J. 1990. Statistical analyses of multi location trials. Advances in Agronomy. 44: 55-85.

Das, S., Misra, R.C., Patnaik, M.C. and Das, S.R. 2010. G × E interaction, adaptability and yield stability of mid-early rice genotypes. Indian Journal of Agricultural Research. 44: 104-111.

Deikman, J., Petracek, M. and Heard, J.E. 2011. Drought tolerance through biotechnology: improving translation from the laboratory to farmers' fields. Current Opinion in Biotechnology, 23: 1-8

Farshadfar, E. and Sutka, J. 2003. Locating QTLs controlling adaptation in wheat using AMMI model. Cereal Research Communications. 31: 249-255. 
Fentie, M., Assefa, A. and Belete, K. 2013. Ammi Analysis of Yield Performance and Stability of Finger Millet Genotypes across Different Environments. World Journal of Agricultural Sciences. 9: 231-237.

Gauch, H.G. and Zobel, R.W. 1996. AMMI analysis of yield trials. In M.S. Kang \& H.G. Gauch. (eds.). Genotype-by-environment interaction. Boca Raton, FL, CRC Press. pp: 85-122.

Gomez, K.A. and Gomez A.A. 1984. Statistical Procedures for Agricultural Research. 2nd ed. John Wiley and Sons, New York.

Hussain, S.S., Kayani, M.A. and Amjad, M. 2011. Transcription factors as tools to engineer enhanced drought stress tolerance in plants. Biotechnology Progress, 27 (2): 297- 306

Ibrahim, M.A. 2001. Application of agro-ecological zones database in drought management and water availability assessment, Bangladesh Agricultural Research Council, Farmgate, Dhaka.

Islam, T. 1996. Utilization of on Farm reservoir for drought alleviation in T. Aman rice in Barind Area. Annual report presented in internal review of Bangladesh Rice Research Institute.

Karim, Z, Ibrahim, A.M., Iqbal, A. and Ahmed, A. 1990. Drought in Bangladesh Agriculture and Irrigations schedules for major crops. Bangladesh Agricultural Research Council. P.10

Kempton, R.A. 1984. The use of biplots in interpreting variety by environment interactions. Journal of Agricultural Science. 103: 123135.

Kulsum, M.U., Hasan, M.J. Akter, A., Rahman, H. and Biswas, P. 2013. Genotype-environment interaction and stability analysis in hybrid rice: an application of additive main effects and multiplicative interaction. Bangladesh Journal of Botany. 42: 73-81.

Misra, R.C., Das, S. and Patnaik, M.C. 2009. AMMI Model Analysis of Stability and Adaptability of Late Duration Finger Millet (Eleusine coracana) Genotypes. World Applied Sciences Journal. 6(12): 1650-1654.

Nadeem, N.M. and Islam, N. 2007. AMMI Analysis of Some Upland cotton genotypes for Yield Stability in Different Milieus. World Journal of Agricultural Sciences. 3(1): 39-44.

Ramamasy, S. and B. Stephan. 2007. MODULE 2: Drought and its impacts. In: Climate variability and change: adaptation to drought in Bangladesh: A resource book and training guide, Electronic Publishing Policy and Support Branch, Communication Division FAO Viale delle Terme di Caracalla, 00153 Rome, Italy.

Turner, N.C. 1979. Drought resistance and adaptation to water deficits in crop plants. In: Mussell H., Staples C. R. (eds). Stress physiology in crop plants. New York, USA, p. 343-372

Yau, S.K. 1995. Regression and AMMI analyses of genotype $\times$ environment Interactions: An empirical comparison. Agronomy Journal. 87: 121-126.

Zobel, R.W., Wright, M.J. and Gauch, H.G. 1988. Statistical analysis of a yield trial. Agronomy Journal. 80: 388-393. 